\title{
Distinctness of perceived three-dimensional form induced by modulated illumination: Effects of certain display and modulation conditions
}

\author{
STEN STURE BERGSTROM, KARL-ARNE GUSTAFSSON, and TORBJÖRN JAKOBSSON \\ University of Umeá, Umeá, Sweden
}

\begin{abstract}
The present study concerns the distinctness of the 3-D shape induced on flat displays by spacemodulated illumination ("shape from shading"). The displays that we used varied in structure, hue contrast, lightness contrast, and in the number of reflectance edges present. The modulations of the illumination were a square-wave grating, a gradual modulation (a blurred grating), and an "O'Brien modulation," which combines an edge and a gradient. The displays were compared, using the paired comparison method, as to the distinctness of the perceived 3-D form. The results showed that the structure and chromatic color of the display were important factors facilitating the distinctness of the induced 3-D shape under all the modulation conditions. The results are discussed in relation to the "vector model" for color constancy, proposed in earlier papers.
\end{abstract}

The effect of shading on perceived three-dimensional (3-D) shape has intrigued perceptionists for a long time. The concave-convex shift when turning a photo of the moon craters upside down is just one example, but it is an interesting one. The first observations of this phenomenon seem to have been made "live" by astronomers who used a new type of telescope that gave an upside-down image of the moon. They complained that the craters looked like bumps, and not craters; a chief astronomer may have been the first to report systematic observations of the phenomenon (Rittenhouse, 1786).

These past observations, which led to the assumption that illumination comes from "above," prompted a major discussion of whether the assumption is inherited or acquired, and some very interesting experiments resulted. Best known are perhaps those by Hess $(1950,1961)$ and Herschberger (1970). Bergström, Gustafsson, and Putaansuu (1984) reported a clear observer preference for perceiving illumination as originating from above by using a Mondrian display illuminated by a square-wave modulated illumination and by comparing the horizontal and vertical orientation of the grating.

In some of the experiments on the effect of the direction of illumination, extra information about depth has been present, for example, that of geometric shape or occlusion, making the conclusions doubtful (see Howard, 1983). In a recent study, Howard, Bergström, and Ohmi (1990) have shown that illumination "from above" should

This study was financially supported by the Swedish Council for Research in the Humanities and Social Sciences by grants to $S$. Bergström under contracts $F$ 470/88 and F 627/89. Address correspondence to $S$. Bergström, Department of Applied Psychology, University of Umeả, Umeå, Sweden. rather be described as "from overhead," because the preferred direction of perceived illumination varies with the orientation of the head; for example, with the head upside down, the preferred direction of illumination is, gravitationally, from below.

A number of highly illustrative demonstrations and experiments with shape from shading have been reported lately (e.g., Ramachandran, 1988; Todd \& Mingolla, 1983), especially in the fields of artificial intelligence and neural networks. Grossberg (1987a, 1987b) has presented a visual-processing theory to explain the synthesizing of 3-D form, color, and brightness perception.

In a series of experiments, Bergström (1982) and Bergström et al. (1984) have studied the effect of display variables on the perceived depth induced by space-modulated illumination. The displays used in those studies were flat, and the illumination was square-wave modulated. The dark phases of the square-wave grating simulated attached shadows, and their observers often reported that the display looked three-dimensionally pleated, like a venetian blind, or even separated in depth at high grating contrasts. Those studies were made within a theoretical frame of reference that stressed the color-constancy aspect of the phenomenon. Perceived (induced) shape from shading means, of course, that the darker areas are perceived as shaded, and not as having a darker color. The perceived depth was considered to be an indication of color constancy.

It has been suggested by one of the present authors (Bergström, 1977, 1982) that Johansson's $(1950,1964)$ perceptual vector analysis of motion could also be applied to reflected light. It is assumed that reflected light is automatically analyzed into a common component corresponding to illumination and relative components corresponding 
to reflectances (in the case of perceived reflectance edges), and/or to the orientations and positions of the reflecting surfaces in space (in the case of perceived illumination edges). It should be stressed here that we are referring to perceived illumination, perceived reflectances, and perceived orientations and positions. This idea is easily understood when applied to chromatic color constancy. If a display consisting of adjacent red and green fields is illuminated by yellow light instead of white, the reflected light from the fields has a common yellowish component that is perceptually connected to the illumination, and the residual components make the fields look red and green instead of orange and yellow-green. The present experiments address the problem of shading, however, and not that of chromatic color constancy. Here, the application of vector analysis is not that simple, and we certainly don't have an algorithm for it.

There are other studies that examine the idea that the retinal image is analyzed into components such as an illumination image (or shading image), a reflectance image, and a 3-D shape (e.g., Barrow \& Tennenbaum, 1978). Gilchrist, Delman, and Jacobsen (1983) discuss an analysis of the retinal image into two component images, one for surface color and one for the pattern of illumination. They also refer to the perception of transparency as another example requiring such an analysis. Adelson and Pentland (1990) introduced a marvelous metaphor, the workshop metaphor, for this type of perceptual analysis.

Bergström et al. (1984) predicted, from the model, that a homogeneous gray display would appear less distinctly pleated than a structured Mondrian display would under the same square-wave space-modulated illumination. Their results are clearly in line with the prediction. $\mathrm{Ob}$ serve that, according to traditional theories on depth perception, the prediction would have been in the opposite direction, because all the geometrical information from the structured Mondrian is about its flatness. The depth information from the modulation of the illumination, however, overrides the contradictory geometrical information. This is contrary to Marr's (1982, p. 248) opinion about the relation between shading and other cues. But why does the homogeneous display not appear pleated as frequently as the Mondrian? According to our model, the reason is that in the homogeneous display there is nothing present to reveal the common component characteristics of the modulated illumination. The very same edges that geometrically inform about the flatness of the display simultaneously reveal the commonality that is the typical characteristic of illumination. It takes variation to reveal invariance!

Our assumption that illumination is a common component has been used by Ramachandran and Rogers (1988), and a very interesting parallel is the assumption of a common component in a model for perceived transparency by Gerbino, Stultiens, Troost, and de Weert (1990). Walraven's (1976) data on the disregarding of background can also be interpreted as an example of such an analysis.
We do not know whether the observed effects of display structure depend on the presence of chromatic color, on lightness or color contrast, or on the presence of color edges. What we do know from control experiments is that a line grating drawn on the homogeneous gray display does not help. Certain effects of edges on color perception have been demonstrated by Krauskopf (1963) and by Yarbus (1967), who made the color border between a central disk and a surrounding ring stationary on the retina. The effect was that the central disk was filled in by the color of the surrounding, indicating that the color of a surface is determined by the edges of the surface. O'Brien (1958) assumed that "perhaps the visual mental apparatus gets the clue from the edge and applies it to the whole area" (p. 116). Land and McCann (1971) introduced the retinex theory, which proposes that the information at the edges of a surface is used to give color constancy, but they disregarded the perception of illumination. Gilchrist et al. (1983) discuss the difference between lightness edges and illumination edges and also the need for an integration of information at the edges (cf. Arend, 1973), in line with the retinex theory, but unlike Land and McCann they stress the fact that illumination is perceived.

In the earliest description of our approach to color constancy (Bergström, 1977), a functional difference between stepwise and gradual luminance changes in space was assumed. The luminance steps were typically assumed to inform about color borders, whereas luminance gradients were assumed to correspond to shadows (i.e., 3-D form). Stepwise illumination edges do of course exist in our environment, and they are sometimes mistaken for reflectance edges. Crossings between illumination edges and reflectance edges help reveal this characteristic. The above assumption about the functional difference between steps and gradients is not a basic assumption in our model, but was originally made just to explain the perceptual phenomena called Craik-Cornsweet-O'Brien effects (see, e.g., Cornsweet, 1970, chap. 9; O'Brien, 1958). Figure 1 illustrates the way to produce such a phenomenon by rotating a black and white circular disk; the luminance distribution is determined by the form of the black and white halves of the disk.

According to the above assumption, the explanation for the O'Brien phenomenon is that the gradual increase in
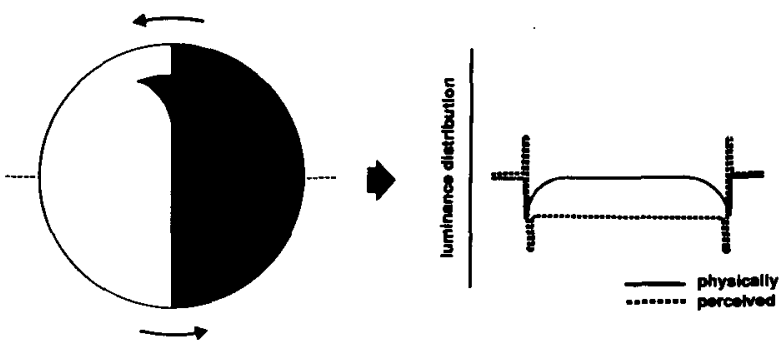

Figure 1. The type of black and white disk that, when rotated, gives the luminance distribution illustrated by the diagram to the right. The dotted line illustrates the perceived luminance. 
luminance inside the step cannot compensate for the decrease at the step, because they have different functions. The luminance step informs about a cliff in depth, provided that its characteristic as an illumination edge is revealed. If not, it informs about a reflectance border. The gradient definitely informs about a 3-D form. This means that the darker central disk should not be perceived as being blacker than the ring, but as being curved in depth and either concave or convex. And if so, the same display variables that helped reveal the illumination characteristics of our square-wave grating should be effective even here in giving the central disk a distinctly perceived 3-D (convex or concave) form, rather than a lower brightness. In her paper, O'Brien (1958) even mentions "a somewhat three-dimensional effect of an inner body sitting in front of the larger outer body" (p.115).

\section{Problems}

In the present paper, four experiments studying perceived depth induced by modulated illumination are reported. The purpose of the experiments was to study the effect of certain display conditions and certain modulation conditions on the distinctness of the induced 3-D shape. The displays were varied in structure, color, and contrast, and in the number of reflectance/color edges. According to the theoretical model, intersections between illumination edges and reflectance edges should reveal the common component characteristics of the illumination edges. This will result in color constancy and in induced 3-D shape. Earlier observations lead us to assume that the contradictory information from the projection of the reflectance edges will be overruled by the information about depth that is inherent in the modulated illumination.

Problem 1. What is the effect of display structure in the form of chromatic color and lightness contrast on the distinctness of the perceived 3-D shape induced by modulated illumination? This problem is addressed in Experiments 1 and 2 by using square Mondrian displays and square-wave space-modulated illumination (Experiment 1), and circular displays and an ' $O$ 'Brien illumination" (Experiment 2). The different modulations will be explained in more detail below.

Problem 2. Is there any effect of the number of reflectance edges in the display (crossed by or parallel to the illumination edges) on the distinctness of the perceived 3-D shape? This problem is addressed by Experiments 3 and 4 by using square Mondrian displays and a squarewave modulated illumination (Experiment 3), and circular displays and an O'Brien illumination (Experiment 4).

Problem 3. Do the same display factors that facilitate the induction of 3-D shape under the sharp grating illumination (illumination edges) also work under a gradient illumination (i.e., a blurred grating, Experiment 1) and under the combination of a sharp edge and a gradient (i.e., an O'Brien illumination, Experiments 2 and 4)?

\section{Illuminations}

The spatial modulations of the illuminations used were the following. In Experiment 1, a square-wave space- modulated illumination and a gradient illumination were used. The gradient illumination was produced by simply defocusing a square-wave grating. In Experiment 3, the same sharp square-wave grating was used as in Experiment 1. Its spatial frequency was $2.2 \mathrm{c} / \mathrm{deg}$ in both experiments. We know from earlier studies that a blurred grating often induces a more compelling 3-D shape than a sharp one does (Bergström, 1982, p. 373), probably because a gradual modulation of the illumination (and consequently of the luminance) is less inclined to be mistaken for a reflectance edge than is a stepwise modulation (cf. the famous ringed shadow experiment by Hering, 1907/ 1964, p. 8).

In Experiments 2 and 4, the modulation of the illumination was very special. It was designed to give an O'Brien phenomenon (O'Brien, 1958), which means that the inner disk of a circular display appears darker (blacker) than the outer ring having the same physical luminance. The phenomenon is accounted for by the luminance profile of the edge between the ring and the disk being a combination of a step and a gradient.

\section{GENERAL METHOD}

In Experiments 1 and 3, Mondrian-type displays were illuminated by square-wave modulated illuminations, and the distinctness of the perceived 3-D shape was studied using displays varying in color, structure, and number of reflectance edges.

In Experiments 2 and 4, circular displays were illuminated by a projector giving an O'Brien illumination, that is, the projector had a black/white slide that was a positive photo of a spinning disk, giving the distribution of illumination that is typically used to demonstrate the O'Brien effect (see Figure 1).

The square Mondrian displays in Experiments 1 and 3 varied as to color, lightness contrasts, and number of reflectance edges, as did the circular displays in Experiments 2 and 4.

The experimental method used in all the experiments was a paired comparison method comparing different displays with regard to the distinctness of the perceived 3-D shape induced by the modulated illuminations. Perceived 3-D shape means that the square Mondrian displays with the superimposed gratings appear pleated in depth, and the central disk of the circular displays with the superimposed O'Brien illumination appears concave or convex.

\section{Apparatus and Procedure}

The stimulus displays were presented two at a time to the subjects, and were illuminated by one of two projectors. The first projector contained a slide of a photographically produced square-wave grating (Experiments 1 and 3) or a slide of two identical O'Brien disks (Experiments 2 and 4). The second projector illuminated the two displays evenly during the pauses (about $10 \mathrm{sec}$ ) between the 4-sec exposures of the grating. Thus, the flat displays were always visible to the subjects and the exposures merely meant that the even illumination was replaced for $4 \mathrm{sec}$ by the square-wave or $O^{\prime} B$ rien illumination. The experimental room was darkened, except for the illuminated stimulus displays. The walls of the room were painted black in order to minimize the amount of scattered light.

In order to minimize the effect of inexact aiming of the projectors, each cardboard display was fixed to the end of a rod sticking out from a background of black velvet. The length of the rods, and thus the distance between the displays and the background, was $80 \mathrm{~cm}$. The rods were not visible to the observer.

Both projectors were hooked to a voltage stabilizer and to variable transformers to make it possible to match the illumination level 
during the pauses to that of the exposures. The luminance levels were adjusted by variable transformers and Wratten ND filters, and were checked by a Spectra Pritchard photometer. The mean luminance of the stimulus display with the superimposed grating was approximately $8.6 \mathrm{~cd} / \mathrm{m}^{2}$ in Experiment 1 , and $3.0 \mathrm{~cd} / \mathrm{m}^{2}$ in Experiment 3. In Experiments 2 and 4, the luminance of the disk and ring was $13.1 \mathrm{~cd} / \mathrm{m}^{2}$, and that of the dark edge was $12.7 \mathrm{~cd} / \mathrm{m}^{2}$, as measured on the photometer.

The two displays in each pair were presented side by side. All the possible pairs of displays (i.e., 15 pairs in Experiments 1 and 2, and 10 pairs in Experiments 3 and 4 ) were used in the experiment. Each pair was presented twice per condition to each subject. The order of presentation was counterbalanced, and the right/left positions of the displays in each pair was different for the two presentations.

The square Mondrian displays in Experiments 1 and 3 had a size of $25 \times 25 \mathrm{~cm}$ corresponding to $3.2^{\circ} \times 3.2^{\circ}$ of visual angle. The circular displays in Experiments 2 and 4 had a diameter of $27 \mathrm{~cm}$ corresponding to $3.9^{\circ}$ of visual angle. The displays were made from NCS color samples, and will be specified in this study by their NCS values (Swedish Standard Institution, 1979a, 1979b). The square Mondrian displays will be identified by capital letters $(A, B, \ldots)$ and the circular displays by Roman numerals (I, II, ...). The viewing distances were $450 \mathrm{~cm}$ in Experiments 1 and 3, and $400 \mathrm{~cm}$ in Experiments 2 and 4 . The subjects were seated, and binocular viewing was allowed.

\section{Subjects}

Six subjects served in Experiments 1 and 2, and 5 served in Experiments 3 and 4 . All of them had normal color vision (Ishihara) and normal visual acuity, with or without correction.

\section{Instructions}

In all the experiments, the subjects were instructed to choose the display in each pair that had the most distinct 3-D shape. The instructions were based on evidence from pilot studies showing that subjects often report the perceived 3-D pleated form of the display as more or less distinct under different conditions. Each experimental session started with a demonstration of a typical display, and the subject was asked to describe what he/she saw. All the subjects spontaneously described 3-D percepts. After this introduction, the subject received the instructions, and a demonstration with 3-D dummies was given to make sure that the experimenter and the subject agreed on the task. It was stressed by the experimenter that there were no right or wrong percepts.

\section{EXPERIMENT 1}

The purpose of Experiment 1 was to compare different flat displays with respect to the distinctness of the 3-D form induced by two different modulations of the illumi-

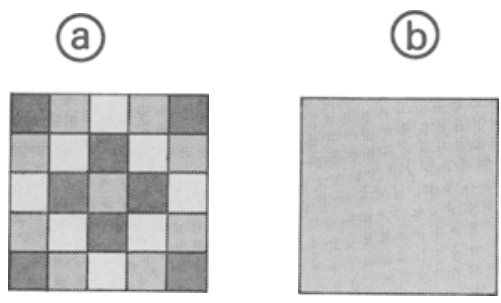

Figure 2. The displays used in Experiment 1: The layout of Displays $A, B, C$, and D (a), and the layout of Displays $E$ and $F(b)$.

nation. The first type of modulation was the optimal square-wave modulation for "pleated" responses, according to earlier studies. The second modulation was a cyclic gradual modulation produced by defocusing a high-contrast square-wave grating. The defocusing was brought about by adding a convex lens to the perfectly focused lens of the projector.

\section{Method}

Apparatus and Procedure. The apparatus and the procedure are described in the General Method section. Two gratings were used. One of them had a contrast of 0.355 , which is optimal for inducing perceived pleated 3-D form, according to the results of earlier experiments. It was used in the "sharp" condition. The other grating, with a contrast of 0.933 , was blurred by adding a +0.30 lens to the focused lens of the projector. The blurring gave a cyclic gradual grating that roughly resembled a sine-wave modulation. The two conditions will hereinafter be called the sharp and the blurred conditions.

The two displays in each pair were presented side by side, in the same (sharp or blurred) modulated illumination.

Displays. Six square $25 \times 25 \mathrm{~cm}$ Mondrian displays were used; the main layout of the displays is illustrated in Figure 2. The displays were compared under both the sharp and blurred conditions. They are specified below with their NCS color coordinates in parentheses.

Display A: A checkered Mondrian display, consisting of 25 squares $(5 \times 5 \mathrm{~cm})$ of three different colors (the NCS values of the color samples were 1030-R90B, 1070-R, and 4040-Y).

Display B: A checkered Mondrian display, consisting of 25 squares of three different colors with minimized lightness contrast (2050-R30B, 4040-Y, and 3040-G).

Display $C$ : A checkered Mondrian display, consisting of 25 squares with three different shades of the same red/purple hue, which were chosen to approximately match the lightness contrasts of Display A (3030-R30B, 2050-R30B, and 3050-R30B).

Table 1

Preference Ratios for the Displays in the Top Row Compared With Those in the Left Column as to the Distinctness of Perceived 3-D Shape for 6 Subjects With Two Judgments Per Pair in the Sharp Condition of Experiment 1

\begin{tabular}{ccccccc}
\hline & \multicolumn{7}{c}{ Display } & & \\
\cline { 3 - 7 } Display & A & B & C & D & E & F \\
\hline A & & $5 / 12$ & $9 / 12$ & $4 / 12$ & $4 / 12$ & $3 / 12$ \\
B & $7 / 12$ & & $7 / 12$ & $4 / 12$ & $3 / 12$ & $4 / 12$ \\
C & $3 / 12$ & $5 / 12$ & & $2 / 12$ & $2 / 12$ & $2 / 12$ \\
D & $8 / 12$ & $8 / 12$ & $10 / 12$ & & $5 / 12$ & $3 / 12$ \\
E & $8 / 12$ & $9 / 12$ & $10 / 12$ & $7 / 12$ & & $3 / 12$ \\
F & $9 / 12$ & $8 / 12$ & $10 / 12$ & $9 / 12$ & $9 / 12$ & \\
Totals & $35 / 60$ & $35 / 60$ & $46 / 60$ & $26 / 60$ & $23 / 60$ & $15 / 60$ \\
\hline
\end{tabular}




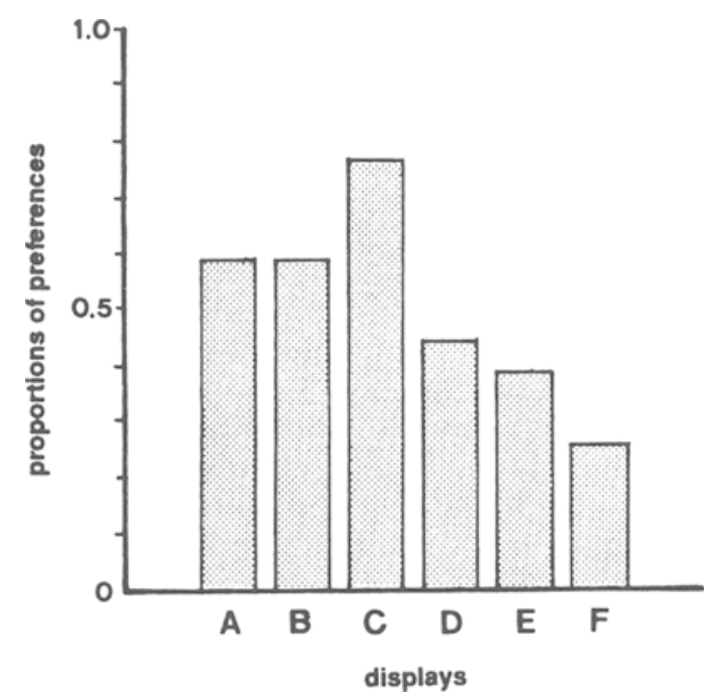

Figure 3. Proportions of preferences for the six displays in the sharp condition of Experiment 1.

Display D: A checkered Mondrian display, consisting of 25 squares with three different shades of gray, giving approximately the same contrasts between squares as in Display A (2502-B, 4502-B, and 5502-B).

Display E: A homogeneous red/purple display; the same reddish color as that of the center square of Display C (2050-R30B).

Display F: A plain gray display; the same gray as that of the center square of Display D (4502-B).

\section{Results and Discussion}

The preferences of displays in the sharp condition are presented in Table 1, indicating the distinctness of the perceived 3-D form of the displays. The sums of each column give a rank order among the displays. The displays are compared with regard to first choices in Figure 3; Display $\mathrm{C}$ is given the highest ranking, and Display $\mathrm{F}$ the lowest.

The corresponding data for the blurred condition are presented in Table 2 and Figure 4. Here, Displays E and F show the lowest rankings, whereas Display $C$ is in the high-ranking group.

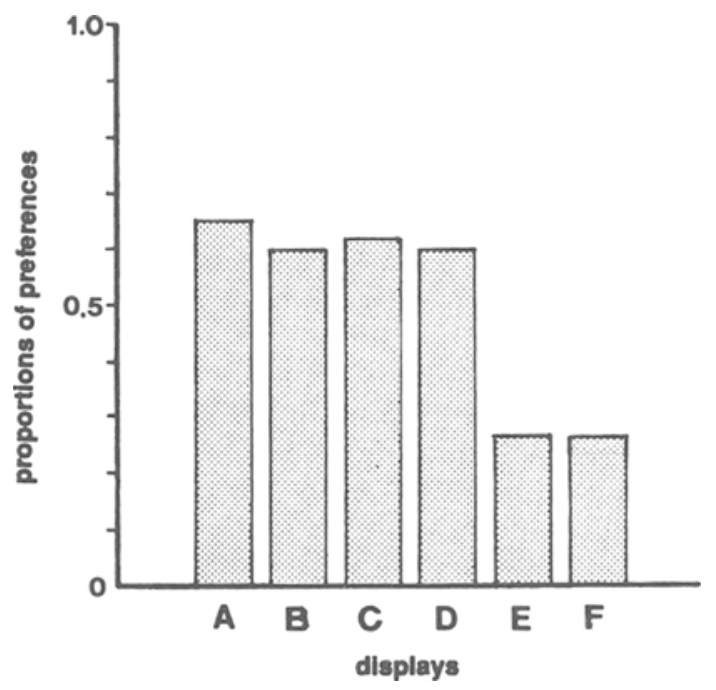

Figure 4. Proportions of preferences for the six displays in the blurred condition of Experiment 1.

When converting the obtained proportions to a scale of distinctness, using Thurstone's law of comparative judgments, we arrive at the scales illustrated in Figure 5. It is obvious from the results that Displays $F, E$, and $D$ give the lowest perceived distinctness of the induced 3-D form in the sharp as well as in the blurred condition. In the blurred condition, though, the induced depth is so distinct that the scale becomes more compressed, and Display D is grouped with Displays B, C, and A. Only the homogeneous displays, $\mathrm{F}$ and $\mathrm{E}$, are low in distinctness. In the sharp condition, the scale is a bit more stretched and the displays are more widely distributed along the scale, probably because the sharp condition is more ambiguous and the display factors (like chromatic color and lightness contrast) are more important. The blurred condition simulates attached shadows in a more unequivocal way than the sharp condition does. This observation can be compared with the still more compelling perception of motion in depth caused by simultaneous modulation of the illumination in space and time, as reported by Bergström (1982).

Table 2

Preference Ratios for the Displays in the Top Row Compared With Those in the Left Column as to the Distinctness of Perceived 3-D Shape for 6 Subjects With Two Judgments Per Pair in the Blurred Condition of Experiment 1

\begin{tabular}{crrrrrr}
\hline & \multicolumn{6}{c}{ Display } \\
\cline { 2 - 7 } Display & A & B & C & D & E & F \\
\hline A & & $6 / 12$ & $4 / 12$ & $5 / 12$ & $4 / 12$ & $2 / 12$ \\
B & $6 / 12$ & & $6 / 12$ & $7 / 12$ & $3 / 12$ & $2 / 12$ \\
C & $8 / 12$ & $6 / 12$ & & $6 / 12$ & $1 / 12$ & $2 / 12$ \\
D & $7 / 12$ & $5 / 12$ & $6 / 12$ & & $2 / 12$ & $4 / 12$ \\
E & $8 / 12$ & $9 / 12$ & $11 / 12$ & $10 / 12$ & & $6 / 12$ \\
F & $10 / 12$ & $10 / 12$ & $10 / 12$ & $8 / 12$ & $6 / 12$ & \\
Totals & $39 / 60$ & $36 / 60$ & $37 / 60$ & $36 / 60$ & $16 / 60$ & $16 / 60$ \\
\hline
\end{tabular}


sharp

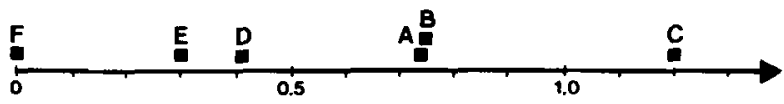

blurred

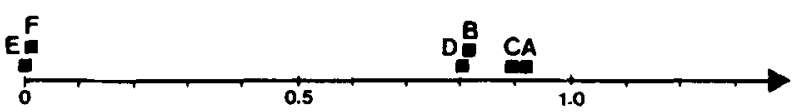

distinctness

Figure 5. The six displays of Experiment 1 plotted on scales of the distinctness of their perceived 3-D shape for the sharp and blurred conditions.
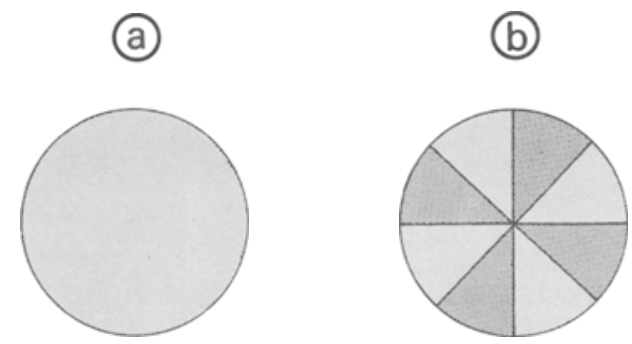

Figure 6. The layout of the displays used in Experiment 2: The homogeneous displays, I and II (a), and the eight-sector displays, III-VI (b).
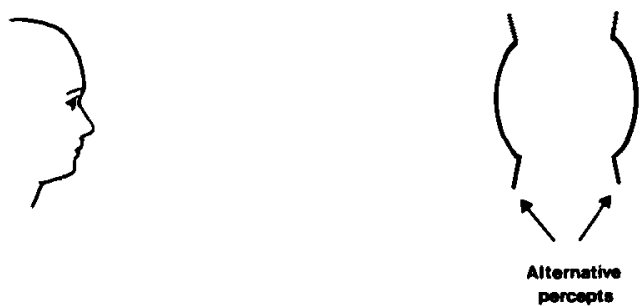

Figure 7. The perceived convex or concave 3-D shape of the $O^{\prime} B$ rien disk, as described by our subjects.

\section{EXPERIMENT 2}

\section{Method}

Displays. In this experiment, flat circular displays were illuminated by an O'Brien illumination. The displays varied in color and lightness contrast in the same way that the Mondrian displays did in Experiment 1. The diameter of the displays was $27 \mathrm{~cm}$. The following displays are shown in Figure 6.

Display I: A homogeneous, monochromatic red display (0050-R). Display II: A homogeneous gray display (3500).

Display III: An eight-sector display, two shades of gray (2500, 4000).

Display IV: An eight-sector display with two chromatic colors, red and blue (2040-R, 0040-R90B).

Display V: An eight-sector display of different lightnesses, but with the same red color (1040-R, 2040-R).

Display VI: An eight-sector display, red and blue, with minimum lightness contrast, that is, the lowest contrast we could achieve using the NCS color samples (0050-R, 1040-R90B).

Procedure. The procedure was as described previously. Before starting the comparisons, there was an instruction session in which displays with the O'Brien illumination were presented to the subject and he/she was asked to report his/her impressions of the displays. As soon as the subject spontaneously reported perceiving a 3-D form, he/she was asked to compare the distinctness of the perceived 3-D form of the two displays. Three-dimensional models of circular concave and convex objects were used to clarify the task. Figure 7 shows the 3-D forms decribed by our subjects.

\section{Results and Discussion}

The preference ratios are given in Table 3 and Figure 8. The highest ratio is for Display IV (89/120), followed by Display VI. This means that the two displays with two chromatic colors presented the most distinct 3-D impression under our specrai $O$ 'Brien illumination. We obtained the lowest ratios for Display II, which is the homogeneous gray display, followed by Display III with two shades of gray. Display I, the homogeneous red one, was preferred to the gray ones. As in our earlier Mondrian studies, induced 3-D form is facilitated by chromatic color (see Gustafsson, 1987). The preference ratios have been transferred not only to rank order between displays, but also to a scale of distinctness using Thurstone's law of comparative judgments, Case V (Torgerson, 1958). The scale is illustrated in Figure 9.

Table 3

Preference Ratios for the Displays in the Top Row Compared With Those in the Left Column as to the Distinctness of Perceived 3-D Shape for 6 Subjects With Four Judgments Per Pair in Experiment 2

\begin{tabular}{|c|c|c|c|c|c|c|}
\hline \multirow[b]{2}{*}{ Display } & \multicolumn{6}{|c|}{ Display } \\
\hline & I & II & III & IV & $\mathrm{V}$ & VI \\
\hline I & & $9 / 24$ & $12 / 24$ & $17 / 24$ & $7 / 24$ & $15 / 24$ \\
\hline II & $15 / 24$ & & $13 / 24$ & $18 / 24$ & $17 / 24$ & $19 / 24$ \\
\hline III & $12 / 24$ & $11 / 24$ & & $23 / 24$ & $12 / 24$ & $17 / 24$ \\
\hline IV & $7 / 24$ & $6 / 24$ & $1 / 24$ & & $5 / 24$ & $12 / 24$ \\
\hline $\mathbf{V}$ & $17 / 24$ & $7 / 24$ & $12 / 24$ & $19 / 24$ & & $17 / 24$ \\
\hline VI & $9 / 24$ & $5 / 24$ & $7 / 24$ & $12 / 24$ & $7 / 24$ & \\
\hline Totals & $60 / 120$ & $38 / 120$ & $45 / 120$ & $89 / 120$ & $48 / 120$ & $80 / 120$ \\
\hline
\end{tabular}




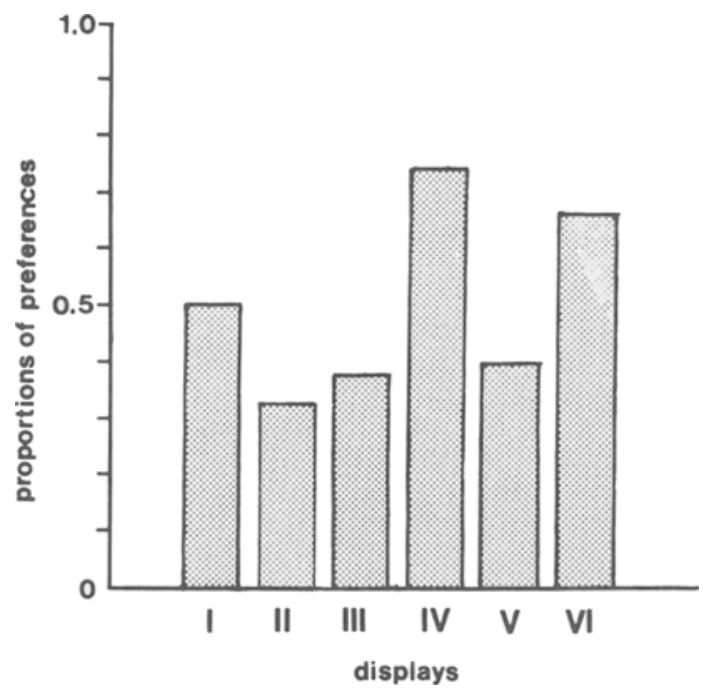

Figure 8. The preference ratios for the six displays of Experiment 2. Paired comparison data for 6 subjects.

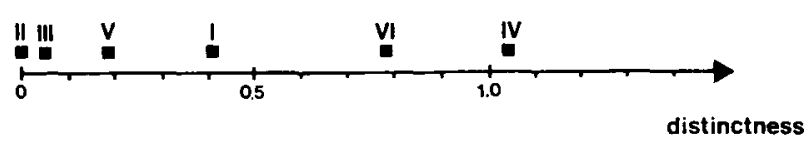

Figure 9. The six displays of Experiment 2 plotted on a scale of the distinctness of their perceived 3-D shape.

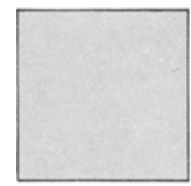

G

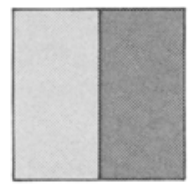

H

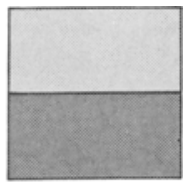

$\mathbf{J}$

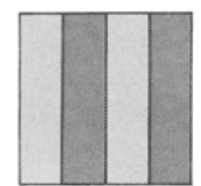

K

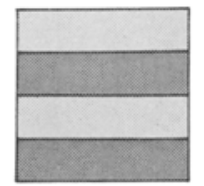

$\mathbf{L}$

Figure 10. The five displays used in Experiment 3. Display G was pink; the others were pink and blue. The superimposed square-wave grating (illumination) was horizontally oriented.

\section{EXPERIMENT 3}

The purpose of Experiment 3 was to study the role played by color borders in inducing an apparent 3-D pleated form on a flat display. Equally sized displays with zero, one, and three reflectance edges, showing crossings or parallelism in relation to the horizontally oriented square-wave modulated illumination, were compared with regard to the distinctness of the induced 3-D pleated form.

\section{Method}

Displays. The following displays were used (see Figure 10). Display G: A homogeneously colored (pink) square (2040-R). Display $H$ : A square, divided vertically into two rectangular halves of different colors (pink and blue, 2040-R, 0040-R90B). One reflectance border is crossed by the horizontal illumination borders of the grating.

Display J: The same two-field display as in Display $\mathbf{H}$, but with the reflectance border horizontally oriented. The reflectance border is parallel to the grating.

Display $K$ : A square, vertically divided into four fields of equal size, with the two colors (2040-R and 0040-R90B) alternating. The reflectance borders are crossed by the illumination borders of the grating.

Display L: The same four-field display as in Display $\mathbf{K}$, but with a horizontal orientation. The three reflectance borders are parallel to the illumination borders.

Procedure. The experimental procedure was the same as that used in Experiment 1. We chose grating contrast 0.355 as the optimal one for inducing perceived pleated 3-D form, according to the results from earlier experiments.

\section{Results and Discussion}

The preference ratios for the displays are tabulated in Table 4; summing the columns gives a rank order of first choices between the displays. The proportions of first choices are shown in Figure 11. The two displays with reflectance borders crossed by the illumination borders of the grating ( $\mathrm{H}$ and $\mathrm{K}$ ) show the highest proportions of first choices, and Display $K$, with three reflectance borders crossed by the illumination borders, shows the very highest proportion.

Thurstone's law of comparative judgments makes it possible to convert our proportions to a scale of distinctness of the induced 3-D form. Using the technique for incomplete matrices (with proportions 0.0 and 1.0 ) proposed by Torgerson (1958), we obtained the scale illustrated in Figure 12.

Table 4

Preference Ratios for the Displays in the Top Row Compared With Those in the Left Column as to the Distinctness of Perceived 3-D Shape for 5 Subjects With Two Judgments per Pair in Experiment 3

\begin{tabular}{ccccrr}
\hline & \multicolumn{5}{c}{ Display } \\
\cline { 2 - 6 } Display & G & H & J & \multicolumn{1}{c}{ K } & \multicolumn{1}{c}{ L } \\
\hline G & & $9 / 10$ & $9 / 10$ & $10 / 10$ & $10 / 10$ \\
H & $1 / 10$ & & $2 / 10$ & $8 / 10$ & $2 / 10$ \\
J & $1 / 10$ & $8 / 10$ & & $10 / 10$ & $8 / 10$ \\
K & $0 / 10$ & $2 / 10$ & $0 / 10$ & & $1 / 10$ \\
L & $0 / 10$ & $8 / 10$ & $2 / 10$ & $9 / 10$ & \\
Totals & $2 / 40$ & $27 / 40$ & $13 / 40$ & $37 / 40$ & $21 / 40$ \\
\hline
\end{tabular}




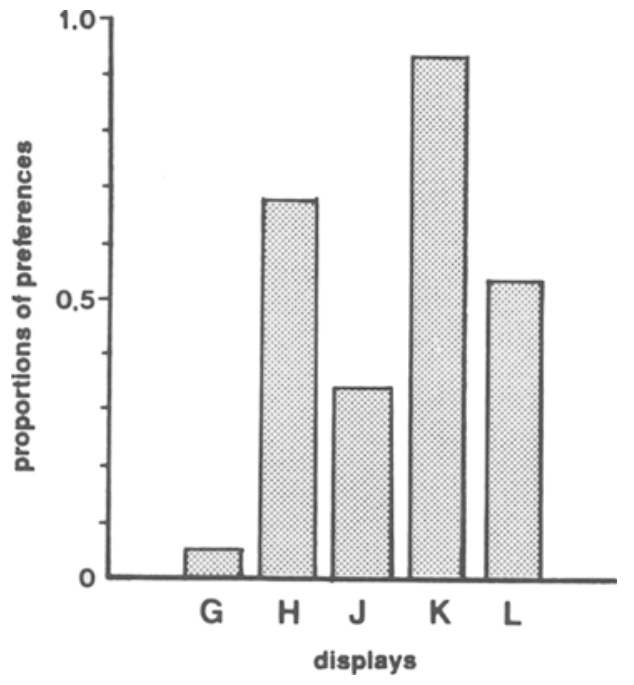

Figure 11. Proportions of preferences for the five displays of Experiment 3.

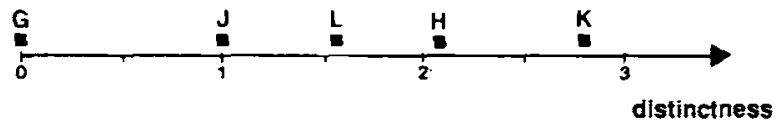

Figure 12. The five displays of Experiment 3 plotted on a scale of the perceived distinctness of their 3-D shape (Thurstone's law of comparative judgments, Case $V$ ).

It is obvious from the results that the number of borders between fields of different colors are of great importance for the distinctness of the induced 3-D form of the displays. The crossings between the illumination borders and reflectance borders in Displays $\mathrm{H}$ and $\mathrm{K}$ give the variation that is needed to reveal the common component characteristics of the grating. According to our own impressions and spontaneous reports from all our subjects, the dark phases of the grating become shadows attached to a pleated object, whereas the light phases appear to be directly illuminated parts of the same object. This is in accordance with our hypotheses based on our theoretical model. It is also obvious that even the number of reflectance borders running parallel to the grating is of some importance, because Display $L$ was preferred to J, which was preferred to $G$. Here there is no variation of color along any single illumination border, but apparently the variation across the whole grating reveals the invariant characteristics of a common illumination, although not as compellingly as the crossed reflectance borders.

\section{EXPERIMENT 4}

\section{Method}

Displays. In Experiment 4, five circular displays were compared as to their tendency to appear concave or convex (i.e., 3-D) under an O'Brien illumination. The purpose of the experiment was to test the hypothesis that the distinctness of the 3-D impression induced by the modulated illumination will increase with the number of reflectance edges intersected by the circular illumination edge. Thus, the number of radial reflectance edges in the display was varied, giving a varied number of sectors within the circular displays. All the displays had chromatic colors; they are described below and are shown in Figure 13.

Display I: A monochromatic red circular display (2040-R); the same display as Display $I$ in Experiment 2.

Display VII: A bipartite display, red and blue (2040-R and 0040-R90B).

Display VIII: A four-sector circular display, with every other sector red and blue.

Display IV: An eight-sector circular display, red and blue; the same display as Display IV in Experiment 2.

Display IX: A 16-sector circular display, red and blue.

\section{Results}

The preference ratios are given in Table 5 and are illustrated in Figure 14. They are clearly in line with our predictions, because the perceived 3-D shape of the flat circular displays increases with the number of intersecting reflectance edges.

The only exception is a small decrease for Display IX compared with Display IV. This difference might be a chance difference, but it might also indicate a tendency toward some sort of optimum regarding number of reflectance edges and size of sectors. The very small sectors, of course, mean shorter length of the illumination edge, the characteristics of which should be revealed by the intersections. For the multisector displays, especially Display IX, our observers sometimes reported a special depth effect-adjacent sectors formed an angle in depth, making the display look like a multiblade propeller. This depth effect may have decreased the perceived distinctness of the concavity/convexity of the central disk of the display.

As in the earlier experiments, the preference ratios have been transferred to a distinctness scale using Thurstone's law of comparative judgments, Case V. This scale is illustrated in Figure 15.
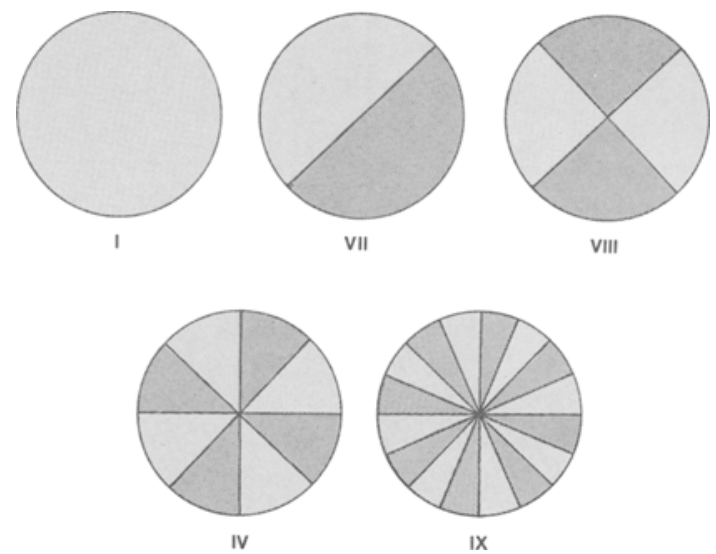

Figure 13. The five displays compared in Experiment 4. The colors were red and blue. 
Table 5

Preference Ratios for the Displays in the Top Row Compared With Those in the Left Column as to the Distinctness of Perceived 3-D Shape for 6 Subjects With Four Judgments Per Pair in Experiment 4.

\begin{tabular}{|c|c|c|c|c|c|}
\hline \multirow[b]{2}{*}{ Display } & \multicolumn{5}{|c|}{ Display } \\
\hline & I & VII & VIII & IV & IX \\
\hline I & & $19 / 24$ & $23 / 24$ & $21 / 24$ & $19 / 24$ \\
\hline VII & $5 / 24$ & & $14 / 24$ & $19 / 24$ & $15 / 24$ \\
\hline VIII & $1 / 24$ & $10 / 24$ & & $17 / 24$ & $17 / 24$ \\
\hline IV & $3 / 24$ & $5 / 24$ & $7 / 24$ & & $13 / 24$ \\
\hline IX & $5 / 24$ & $9 / 24$ & $7 / 24$ & $11 / 24$ & \\
\hline Totals & $14 / 96$ & $43 / 96$ & $51 / 96$ & $68 / 96$ & $64 / 96$ \\
\hline
\end{tabular}

\section{GENERAL DISCUSSION}

The results of Experiments 1 and 2 clearly show an effect of display structure on the perceived distinctness of the induced 3-D form. The homogeneous displays ( $E$ and F) of Experiment 1 were given the lowest ranking in both the sharp and the blurred conditions. In Experiment 2, the eight-sector, two-color displays (IV and VI) had the highest rankings, and the gray and monochromatic ones had the lowest. Even in displays with no structure, the presence of a chromatic color seems to facilitate induced depth; the homogeneous red displays were given some-

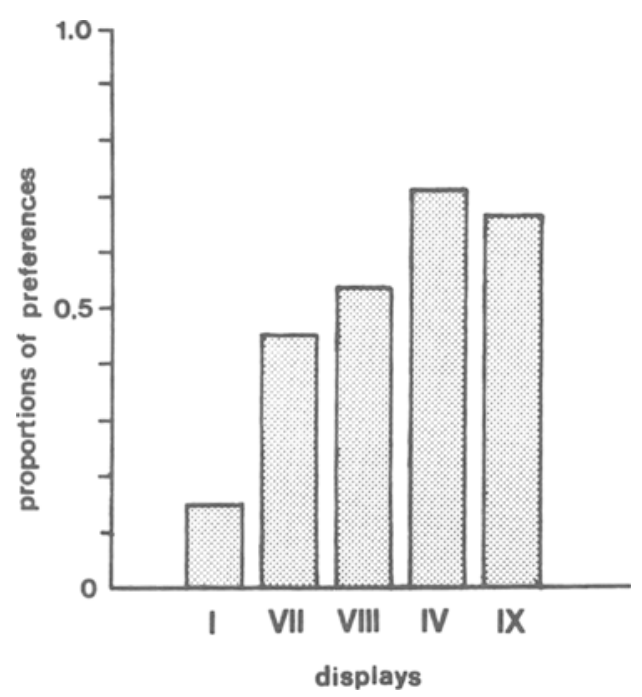

Figure 14. The preference ratios for the five displays of Experiment 4, using paired comparison data.

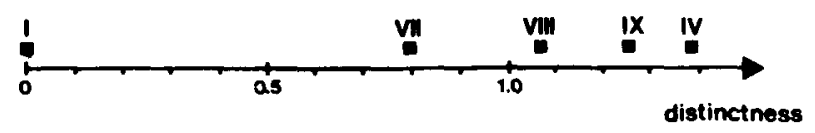

Figure 15. The five displays of Experiment 4 plotted on a scale of the distinctness of their perceived 3-D shape. what higher rankings than the homogeneous gray ones. This is in line with earlier results (Gustafsson, 1987).

Experiments 3 and 4 show an effect of the number of reflectance edges crossed by the illumination edge(s) or even parallel to them. It is also obvious that the same display factors that influenced the distinctness of the 3-D form in the sharp grating condition were also effective when the illumination border(s) were gradients (Experiment 1 ) or a combination of an edge and a gradient (the O'Brien illuminations of Experiments 2 and 4).

The results confirm earlier observations that the perceived 3-D shape induced by spatial modulation of the illumination of a flat display is much more distinct for structured than for homogeneous displays (Bergström et al., 1984). According to traditional theories of depth perception, the geometric projection of the structure of the displays (e.g., the straight and parallel reflectance edges) should inform about their flatness. The homogeneous displays should be more vulnerable to the shapeinducing effect of shading. Both our present and earlier observations contrast with this. They are in line, however, with our theoretical model, which assumes that spatial modulation of illumination always informs about the 3-D layout, provided that the modulation is not mistaken for a reflectance gradient. And the very structure of the display, the projection of which should inform about its flatness, is what reveals the illumination characteristics of our illumination gradient. That is why structure increases the distinctness of the induced depth.

But why, then, is there an effect of increasing degree of structure (e.g., number of reflectances, reflectance edges, etc.)? The reason, probably, is that increasing reflectance variation within the display increases the range of conditions under which the characteristics of the illumination edges are invariant, Again, it takes variation to reveal invariances. The same might not hold for the conflicting information from the geometric projection, that is, the information about the flatness of our displays. The lack of divergence and convergence in the projection of the distally parallel lines in our Mondrian displays does not necessarily become stronger or more effective just because the lines themselves are more distinct. But bigger differences between the quadrangles of the Mondrian do increase the variation within which the contrasts of our 
illumination edges are invariant. So, increasing the degree of structure of the display increases the chances of revealing the invariances of the illumination edges without simultaneously increasing the geometric information about flatness.

The same explanation might also hold for the effect of the number of reflectance edges. Even when parallel to the illumination edges of the grating, the number of reflectance edges is important. They do not increase the variation along any single illumination edge, but the invariance of the grating is repeated over the different surface colors of the display. Thus, the effect of number of reflectance edges, even if not crossed by illumination edges, strongly supports our assumption that the proximal stimulus is analyzed into common and specific components, and that commonality is the feature that makes it possible for our perceptual system to distinguish spatial variation of illumination from that of reflectance. And this possibility is, of course, a prerequisite for color constancy. Our results on the O'Brien effect also support the assumption that there is a functional difference between edges and gradients (cf. Gilchrist et al.'s 1983 “edge classification").

\section{REFERENCES}

Adelson, E. H., \& Pentland, A. P. (1990). The perception of shading and reflectance (Vision and Modeling Tech. Rep. No. 140). Cambridge, MA: MIT Media Laboratory

AREND, L. E. (1973). Spatial differential and integral operations in human vision: Implications of stabilized retinal image fading. Psychological Review, 80, 374-395.

BarRow, H. B., Tennenbaum, J. M. (1978). Recovering intrinsic scene characteristics from images. In A. R. Hanson \& E. M. Riseman (Eds.), Computer vision systems (pp. 3-26). New York: Academic Press.

BERGSTRÖM, S. S. (1977). Common and relative components of reflected light as information about the illumination, colour, and threedimensional form of objects. Scandinavian Journal of Psychology, 18, 180-186.

Bergström, S. S. (1982). Illumination, color, and three-dimensional form. In J. Beck (Ed.), Organization and representation in perception (chap. 18, pp. 365-378). Hillsdale, NJ: Erlbaum.

Bergström, S. S., Gustafsson, K.-A., \& PutaansuU, J. (1984). Information about three-dimensional shape and direction of illumination in a square-wave grating. Perception, 13, 129-140.

CoRnsweet, T. N. (1970). Visual perception. New York: Academic Press.

Gerbino, W., Stultiens, C. I. F. H. J., Troost, J. M., \& De Weert, C. M. M. (1990). Transparent layer constancy. Journal of Experimental Psychology: Human Perception \& Performance, 16, 3-20.

Gilchrist, A. L., Delman, S., Jaconsen, A. (1983). The classification and integration of edges as critical to the perception of reflectance and illumination. Perception \& Psychophysics, 33, 425-436.

GrossBerg, S. (1987a). Cortical dynamics of three-dimensional form, color, and brightness perception: I. Monocular theory. Perception \& Psychophysics, 41, 87-116.

GrossBerG, S. (1987b). Cortical dynamics of three-dimensional form, color, and brightness perception: II. Binocular theory. Perception \& Psychophysics, 41, 117-158.

Gustafsson, K.-A. (1987). Perceived three-dimensional form induced by modulated illumination (DAPS Rep. No. 21). Umeá, Sweden: University of Umeá.

Hering, E. (1964). Outlines of a theory of the light sense (L. M. Hurvich and D. Jameson, Trans.). Cambridge, MA: Harvard University Press. (Original work published 1907)

HERSCHBERGER, W. (1970). Attached-shadow orientation perceived as depth by chickens reared in an environment illuminated from below. Joumal of Comparative \& Physiological Psychology, 73, 407-411.

Hess, E. H. (1950). Development of the chick's response to light and shade cues to depth. Journal of Comparative \& Physiological Psychology, 43, 112-122.

Hess, E. H. (1961). Shadows and depth perception. Scientific American, 204, 139-148.

HowARD, I. P. (1983). Occluding edges in apparent reversal of convexity and concavity. Perception, 12, 85-86.

Howard, I. P., Bergström, S. S., \& OHMI, M. (1990). Shape from shading in different frames of reference. Perception, 19, 523-530.

Johansson, G. (1950). Configurations in event perception. Uppsala: Almqvist \& Wiksell.

Johansson, G. (1964). Perception of motion and changing form. Scandinavian Journal of Psychology, 5, 181-208.

KRAUSKopf, J. (1963). Effect of retinal image stabilization on the appearance of heterochromatic targets. Journal of the Optical Society of America, 53, 741-744.

LAND, E. H., MCCANN, J. J. (1971). Lightness and the retinex theory. Journal of the Optical Society of America, 61, 1-11.

MARR, D. (1982). Vision. San Francisco: Freeman.

O'BRIEN, V. (1958). Contour perception, illusion and reality. Journal of the Optical Society of America, 48, 112-119.

Ramachandran, V. S. (1988). Perception of shape from shading. $\mathrm{Na}$ ture, 331, 163-166.

Ramachandran, V. S., \& Rogers, D. (1988). Perception of shape from shading. Perception, 17, 360. (11th ECVP Abstracts p. A22)

RITTENhouse, D. (1786). Explanation of an optical deception. Transactions of the American Philosophical Society, 2, 37-42.

SWEDISH STANDARD INSTITUTION (1979a). Colour atlas (Swedish Standard SS 0191 02). Stockholm: Author.

SWedish Standard Institution (1979b). Colour notation system (Swedish Standard SS 0191 00). Stockholm: Author.

TodD, J. T., \& Mingolla, E. (1983). Perception of surface curvature and direction of illumination from patterns of shading. Journal of Experimental Psychology: Human Perception \& Performance, 9, 583-595.

TORGERSON, W. S. (1958). Theory and methods of scaling. New York: Wiley.

WALRAVEN, J. (1976). Discounting the background: The missing link in the explanation of chromatic induction. Vision Research, 16, 289-295.

YARBUS, A. L. (1967). Eye movements and vision. New York: Plenum.

(Manuscript received February 22, 1991; revision accepted for publication November 18, 1992.) 